\title{
ANÁLISIS LEXICOMÉTRICO DE LA ESPECIFICIDAD DE LA ESCRITURA DIGITAL DEL ADOLESCENTE EN WHATSAPP*
}

\section{LEXICOMETRIC ANALYSIS OF THE SPECIFICITY OF TEENAGERS' DIGITAL WRITING IN WHATSAPP}

\author{
DR. ESTEBAN VÁZQUEZ-CANO \\ Facultad de Educación. Dpto. Didáctica, Organización Escolar y Didácticas Especiales. \\ Universidad Nacional de Educación a Distancia (UNED). Madrid (España) \\ evazquez@edu.uned.es

\section{DR. SANTIAGO MENGUAL-ANDRÉS} \\ Facultad de Filosofía y Ciencias de la Educación. Dpto. Educación Comparada e \\ Historia de la Educación. Universidad de Valencia (España) \\ santiago.mengual@uv.es
}

\section{DRA. ROSABEL ROIG-VILA}

Facultad de Educación. Dpto. Didáctica General y Didácticas Específicas. Universidad de Alicante (España)

rosabel.roig@ua.es

\section{RESUMEN}

El artículo presenta una investigación en la que se analizan, desde una perspectiva lexicométrica y factorial, los aspectos lingüísticos y paralingüísticos más relevantes de la escritura digital síncrona del adolescente español, en uno de los programas de mensajería instantánea más utilizados en la actualidad (WhatsApp@). La escritura en soportes digitales móviles (smartphones y tabletas) es una de las actividades más realizadas en nuestra sociedad y constituye un componente esencial de la competencia comunicativa en la Sociedad de la Información. La comunicación digital forma parte de nuestras vidas y el análisis del uso comunicativo digital y ubicuo con dispositivos y programas tiene amplias repercusiones sociales, lingüísticas y pedagógicas. La investigación se ha contextualizado en una muestra de 417 conversaciones de WhatsApp de estudiantes de enseñanza secundaria, de entre 13 y 16 años, en cuatro provincias españolas. La metodología de investigación ha sido de corte cuantitativa para abordar el análisis lexicométrico del corpus lingüístico-digital con refe-

\footnotetext{
* Este trabajo se enmarca en el Proyecto de la Dirección General de Investigación y Gestión del Plan Nacional I+D+I del Ministerio de Educación de España (Aprendizaje ubicuo con dispositivos móviles: elaboración y desarrollo de un mapa de competencias en educación superior) EDU201017420-Subprograma EDUC.
} 
rencia a los elementos lingüísticos y paralingüísticos más relevantes; para, posteriormente, realizar el análisis de las correlaciones entre diferentes variables independientes que expliquen patrones lingüísticos y de uso en la escritura digital. Los resultados muestran que la escritura digital en este tipo de programas tiene una serie de características específicas ortotipográficas y audiovisuales condicionadas por variables de uso, el tamaño de la pantalla del dispositivo, la horas de conversación y la relación establecida entre los interlocutores.

Palabras clave: Escritura digital, lexicometría, especificidad léxica, WhatsApp, ciberlingüística.

\section{ABSTRACT}

The article presents a lexicometric and factorial research in which we analyze the most relevant linguistic and paralinguistic characteristics of Spanish teenagers' digital writing in one of the most popular and used instant messaging programs (WhatsApp@). Writing in digital mobile devices (smartphones and tablets) is one of the most commonly activities in our society and is an essential component of communicative competence in the Information and Communication Society. Digital communication is part of our lives and the analysis of the use of digital and ubiquitous communication devices and programs has a broad social, language, and teaching impact. The research has been contextualized in four Spanish provinces with a sample of 417 WhatsApp digital chats among high school students aged from 13 to 16. A quantitative methodology has been implemented from a lexicometric approach in order to analyze the digital linguistic corpus with reference to the most relevant linguistic and paralinguistic elements. Therefore, we have established correlations between independent variables that could explain digital writing linguistic patterns and usage. The results show that digital writing in this instant messaging app has special orthotypographic and audiovisual characteristics conditioned by usage variables such as size of the device display, hours of conversation, and relationship between speakers.

Keywords: Digital writing, lexicometry, lexical specificity, WhatsApp, ciberlinguistics.

Recibido: 13.08.2014. Aceptado: 15.03.2015.

\section{INTRODUCCIÓN}

- 13 de diciembre de 1992 fue enviado el primer SMS de la historia. Desde Cesa fecha, el uso del teléfono móvil y su capacidad para remitir mensajes de texto ha evolucionado considerablemente. La irrupción del mensaje corto digital llevó aparejada, en su momento, una gran cantidad de estudios y considerables polémicas sobre la incidencia de este tipo de escritura oralizada en la ortografía del adolescente (Fernández Pinto, 2001; Rivière, 2002; Cassany, 2003; Bodomo y Lee, 2004; Betti, 2006). Pero estas investigaciones realizadas en el pasado sobre escritura de SMS no son directamente aplicables al actual contexto de escritura 
digital con los programas que no limitan el espacio a un número limitado de caracteres y no cargan un coste económico por cada mensaje (García y Monferrer, 2009; Drouin, 2011; González-Lloret, 2011). Por lo tanto, partiendo de estas investigaciones y teniendo en cuenta que los parámetros y condicionantes han variado, se precisan nuevos estudios que delimiten las características fundamentales de la escritura digital desde los principios de ubicuidad, portabilidad y gratuidad (Vázquez-Cano, 2012; Herring, 2013a, b; Bernicot, Goumi, Bert-Erboul, Volckaert-Legrier, 2014).

Hoy en día el concepto de escritura digital es poliédrico, con multitud de situaciones y dispositivos en los que materializarla y en múltiples contextos digitales de carácter personal, académico y profesional en los que el ciudadano debe desenvolverse. Uno de los contextos en el que el uso de la escritura digital es más profuso, es el uso del teléfono inteligente (smartphone) con pantalla táctil para conversar o interactuar socialmente de forma casi constante en la vida diaria, a través de programas de mensajería instantánea (e.g. WhatsApp, Line, etc.), en programas de microblogging (e.g. Twitter) o la aplicación de chat de las redes sociales (e.g. Facebook). La interacción tecnológica ha generado una escritura de la ubicuidad que precisa de análisis rigurosos que permitan observar las prácticas, errores y usos más comunes de esta forma de comunicación, de manera que se pueda contemplar su incidencia en tres ámbitos prioritarios de la lengua: su incidencia social, su estructura lingüística y la metodología de enseñanza para promover una cada vez mayor competencia lingüística (Plester, Wood y Bell, 2008; Parrilla, 2008; Yus, 2011). "WhatsApp" es la aplicación de mensajería instantánea con mayor penetración en el mercado. En septiembre de 2014 cuenta con más de 600 millones de usuarios que envían más de 64.000 millones de mensajes diarios; por lo que se ha convertido en un instrumento de comunicación con gran repercusión social, sobre todo entre los adolescentes (Fundación Telefónica, 2014).

Se presenta en este artículo una investigación en la que analizamos, a través de una metodología cuantitativa, la caracterización de la escritura digital del adolescente en WhatsApp desde el principio de espontaneidad en la muestra y la incidencia de seis variables que afectan al desarrollo de la escritura digital en estos dispositivos: sexo, edad, tamaño de la pantalla en pulgadas, ubicación de la conversación, horas de conversación y relación entre interlocutores (familia-amigos).

\section{LA ESCRITURA DIGITAL DESDE LOS PARÁMETROS DE LA UBICUIDAD, PORTABILIDAD Y GRATUIDAD}

Los nuevos dispositivos digitales móviles (teléfonos inteligentes y tabletas, princi-

palmente) se caracterizan por su reducido tamaño y la posibilidad de ser utilizados en múltiples situaciones y desde cualquier ubicación gracias a su doble conexión 
inalámbrica y de datos. Asimismo, los desarrolladores de aplicaciones móviles (apps) relacionadas con la comunicación y las redes sociales han desarrollado programas de mensajería y chat que posibilitan la comunicación mediante escritura digital síncrona, sin limitación de caracteres y sin coste por cada mensaje emitido (sino mediante pago general con tarifa plana por tráfico de datos GPRS-3G-4G). Esta característica permite el acceso a gran cantidad de contenido audiovisual, que enriquece la conversación digital y que aparece como uno de los condicionantes inexistentes en los anteriores estudios de escritura en SMS (Cassany, 2003; Bodomo y Lee, 2004; Betti, 2006). Otra característica determinante de estos nuevos dispositivos es que la escritura digital se materializa principalmente mediante pantalla táctil (sin teclas físicas que presionar como hace unos años). El dispositivo más usado y con mayor penetración social entre los adolescentes y público general es el smartphone táctil con conexión wifi y tecnología 3-4G.

En el actual contexto mediático adjetivado desde la portabilidad y ubicuidad, los dispositivos digitales móviles se incorporan como instrumentos capaces de promover relaciones entre personas y equipos, y se han convertido en áreas de flujo entre las personas conectadas a la red (Castells, 2007: 267). La comunicación móvil se extiende y está fortaleciendo la plataforma tecnológica de la sociedad en red, una sociedad cuya estructura y hábitos sociales están organizados alrededor de redes microelectrónicas de información y comunicación (Flores, 2009; Bañon, 2010). Para Castells (2009:107) la comunicación inalámbrica se ha convertido en una plataforma de difusión para muy distintos tipos de productos digitalizados, como juegos, música, imágenes y noticias. El estudio de la comunicación móvil, por lo tanto, se puede realizar desde diferentes áreas de conocimiento: sociología, comunicación, cibercultura, educación y lingüística, entre otros (Pinar y Albentosa, 2001; Baron, Squires, Tench y Thompson, 2005; Kamran, 2010; Yus, 2011; Kuteeva, 2011).

En este contexto virtualizado y ubicuo con el empleo de dispositivos móviles, la interacción y la comunicación digital se hacen mucho más acusadas entre adolescentes mediante el uso de los programas gratuitos de mensajería tipo "WhatsApp" (Alonso y Perea, 2008; Andrade, 2008; Elvis, 2009; Kemp, 2010). En el último informe presentado por la Fundación Telefónica (2014) se destaca la predilección de los españoles por enviar mensajes instantáneos antes incluso que realizar una llamada telefónica. Tanto es así que un 96\% de los usuarios encuestados prefiere utilizar alguna aplicación de mensajería antes que descolgar el teléfono. Hay que tener en cuenta que existen 23 millones de usuarios activos de aplicaciones en España; un país que sigue teniendo el récord europeo en tasa de penetración de smartphones con un $66 \%$. En este contexto mediado por la mensajería digital, hay que destacar la fuerza de las herramientas de mensajería. Así, el 95,3\% de los usuarios afirma utilizarlas diariamente, y el 38,1\% afirma consultarlas varias veces cada hora (Fundación Telefónica, 2014). Datos que no hacen más que reflejar la constante actividad 
de los usuarios y la influencia de estas herramientas en la comunicación.

Investigaciones precedentes enfocadas en la caracterización de la escritura en SMS (Betti, 2006; Alonso y Perea, 2008; Andrade, 2008) realizan un enfoque descriptivo, en el que se analiza la caracterización lingüística y ortotipográfica de la escritura digital, en un contexto digital y social que ha variado sustancialmente en los últimos tres años con la irrupción e incidencia de variables contextuales, que condicionan la escritura digital ubicua como son: el "microblogging", la conversación digital síncrona y ubicua sin coste por mensaje, la conversación en grupo y los nuevos dispositivos digitales móviles táctiles y altamente interactivos en lo que se refiere a intercambio de imagen, video y sonido.

La expresión escrita en los nuevos dispositivos digitales móviles con fundamento en la conversación digital síncrona sin coste por mensaje, se fundamenta en que estos modos de escritura buscan recrear la agilidad e informalidad de una charla entre amigos. En el intercambio digital, síncrono y ubicuo con un dispositivo móvil no se controla la narración sino la interacción (Bernicot et al., 2014). La autoría es compartida, pues las sucesivas intervenciones de los interlocutores son las que construyen la continuidad del relato, que acostumbra a ser improvisado y sin aspiraciones estéticas, aunque no por ello insignificante. Este tipo de comunicación digital, facilitada por el contexto cibermediático descrito, está trasladando al registro escrito características típicas de un registro oral porque se intenta que la escritura sea lo más parecida posible a una conversación, en ocasiones, en formato de "grupo de amigos" como se ha apuntado en la literatura científica: "texto escrito oralizado" (Gómez-Torrego, 2001; Faulkner y Culwin, 2005; Crystal, 2008), de "escritura oralizada" (Fortunati, 2001; Gómez Camacho, 2007; Dresner y Herring, 2012), de "oralidad escrita" (Jaffe y Walton, 2000; Hutchby y Tanna, 2008) o de "conversaciones escritas" (Nastri, Peña y Hancock, 2006; Fraca de Barrera, 2006).

Las conversaciones digitales ubicuas y móviles demandan interacción y reciprocidad de igual forma que en una conversación "cara a cara": en ambas la simultaneidad es la característica dominante (Horstmanshof y Power, 2005; Lewis y Fabos, 2005; Gelernter y Mushegian, 2011; Riordan, Markman y Stewart, 2013). Así, los códigos escritos se han de aligerar, empleándose abreviaturas o contracciones y reduciéndose las palabras a una sola letra, a menudo, sin ninguna vocal (Mayans i Planells, 2002; García Galera, 2008; McWilliam, Schepman y Rodway, 2009; Plester, Wood y Joshi, 2009). Este fenómeno ocurre de manera parecida en la expresión oral de cualquier lengua, ya que el sistema lingüístico lo permite por el principio de economía (relajamiento fonético). Además, este nuevo discurso digital escrito se arropa de los principios de la comunicación audiovisual, alterando la representación de emociones, sensaciones, onomatopeyas y los elementos suprasegmentales del discurso escrito por lo icónico: emoticonos, "stickers" (figuras y objetos diversos) y "memes" (fotos con texto generalmente con intención irónica o humorística), animaciones, la integración de video, foto, audio y enlaces en la 
conversación digital; lo que le confiere un alto poder significativo e interpretativo que supera en muchos casos la conversación oral. Estas estrategias favorecen la comprensión del mensaje al posibilitar "la metarrepresentación de las intenciones" que generaron el enunciado (Rosen, Chang, Erwin, Carrier y Cheever, 2010; Lee, 2011; Treurniet y Sanders, 2012; Markman, 2013).

Este nuevo contexto digital que condiciona la escritura en dispositivos móviles afecta a las formas de comunicación social, a los efectos en la escritura de las diferentes lenguas y, por extensión, a la forma de enseñar el uso de la lengua en diferentes contextos y registros que mejoren en el alumnado su competencia lingüística. Las implicaciones sociales se entienden desde los parámetros de la comunicación digital social y su incidencia en el comportamiento humano, su sociabilidad presencial y virtual y las repercusiones que esto está teniendo en las relaciones personales, académicas y profesionales. Las implicaciones lingüísticas afectan a diferentes planos de la lengua: ortotipográfico, morfológico, sintáctico, semántico y pragmático.

El análisis de la escritura digital tiene profundas repercusiones en la sociolingüística y en los parámetros de la Sociedad de la Información y la Comunicación. El estudio de la escritura digital puede explicar la sincronía del idioma, en constante adaptación a los medios digitales, y delimitar sus características más relevantes para así poder entender su uso en los diferentes contextos. No creemos, a priori, que el alumnado vea modificada sustancialmente su escritura en otros registros por el efecto de las conversaciones digitales ubicuas como se está demostrando en otros estudios (Bernicot et al., 2014), pero el entendimiento de éstas y su enseñanza en la educación secundaria deberían servir para favorecer un mejor desarrollo de los usos lingüísticos en diferentes registros y situaciones, y así, facilitar una mayor competencia lingüística en el medio digital.

Este tipo de escritura forma ya parte de un nuevo paradigma narrativo virtual y parece que ya no tendrá marcha atrás. Cada vez más la evolución tecnológica supondrá una mayor interacción y facilidad en la comunicación digital escrita, lo que sin duda tendrá amplias implicaciones sociales, lingüísticas y pedagógicas en el futuro. En esta investigación hemos centrado exclusivamente nuestro análisis en la caracterización lingüística y paralingüística de la escritura digital ubicua.

\section{METODOLOGÍA}

La metodología de la investigación empleada se basa en los enfoques lexicométricos (Etxeberría, García, Gil y Rodríguez, 1995:145) con la aplicación del Análisis Estadístico de Datos Textuales (ADT) que tiene ya una larga tradición en las técnicas estadísticas desarrolladas por la "Escuela Francesa de Análisis de Da-

tos” (Benzécri, 1973, 1979). Entendemos, pues, el análisis estadístico de un texto 
como aquellos procedimientos que, mediante el cómputo de las ocurrencias de una o varias unidades verbales básicas, permiten realizar, a partir de los resultados obtenidos, algún tipo de cálculo, "de reorganizaciones formales de una secuencia textual y análisis estadísticos con el vocabulario resultante de una segmentación". Dichos análisis se conocen generalmente con el nombre de "Lexicometría".

Para su aplicación hemos desarrollado un enfoque eminentemente cuantitativo con un doble objetivo que nos permitiera realizar un análisis de frecuencias previo con cálculos hipergeométricos de especificidad, para posteriormente codificar los datos y proceder al desarrollo del análisis factorial por medio del análisis de correlaciones (Larson-Hall, 2010); y la propuesta de un modelo de regresión lineal multivariante que permita observar qué variables explican con mayor consistencia los usos lingüísticos, paralingüísticos y los elementos gráficos y audiovisuales de la escritura digital en WhatsApp.

\subsection{Objetivos}

Los objetivos de la investigación se centraron en analizar la escritura digital síncrona del adolescente en el programa WhatsApp, tomando en consideración las siguientes dimensiones:

- Establecer las principales características y variaciones ortotipográficas.

- Delimitar la frecuencia y uso de los elementos gráficos con interpretación suprasegmental (iconos, emoticonos y "stickers") y los elementos audiovisuales en la escritura digital.

- Analizar las correlaciones que explican las dos primeras dimensiones atendiendo a la interrelación de seis variables independientes: sexo, edad, tamaño de la pantalla del dispositivo, horas de conversación y relación entre interlocutores.

\subsection{Muestra}

La investigación se ha contextualizado en cinco centros de enseñanza secundaria españoles correspondientes a cuatro provincias diferentes (Madrid, Badajoz, Toledo y Albacete). El procedimiento de recogida de datos conllevó cierta dificultad al ser adolescentes menores de edad y recabar información sujeta a la Ley Española de Protección de Datos. El total de conversaciones digitales síncronas analizadas fue 417, con un cómputo total de 101.401 palabras. Las muestras recabadas fueron obtenidas por ofrecimiento voluntario de los alumnos/as y se obtuvieron sin identificación de los nombres de los interlocutores presentes en las conversaciones, 
asociando un número en clave para su procesamiento y así preservar la identidad de los interlocutores en la conversación. Cada conversación correspondía a un acto comunicativo digital real sin intervención ni conocimiento previo de los colaboradores ni de las variables independientes de análisis posterior. Cada muestra obtenida correspondía a una conversación con una media de 20 intervenciones dialógicas digitales y doce palabras de media por intervención y fue complementada con una ficha de datos en la que figuraban los siguientes ítemes: edad, sexo, tamaño de la pantalla en pulgadas del smartphone, lugar en el que se mantuvo la conversación, relación entre interlocutores (familia o amigos) y tiempo medio dedicado a conversaciones de WhatsApp al día (más o menos de una hora). La muestra obtenida se puede ver en la Tabla I:

Tabla I. Muestra participante por edades y sexo.

\begin{tabular}{|c|c|c|}
\hline Muestra & $\%$ Casos & No \\
\hline Alumnos edad $13 / 15$ & $24,4 \%$ & 102 \\
\hline Alumnos edad 16/17 & $24,7 \%$ & 103 \\
\hline Alumnas edad 13/15 & $24,9 \%$ & 104 \\
\hline Alumnas edad 16/17 & $25,8 \%$ & 108 \\
\hline TOTAL & 100 & 417 \\
\hline
\end{tabular}

\subsection{Técnicas e instrumentos de análisis}

En la investigación lingüística con aplicación de análisis estadístico existen diferentes aproximaciones centradas en el uso de programas de análisis de textos (Lebart y Salem, 1994; Pennebaker, Booth y Francis, 2007). Hemos recurrido a una versión modificada y adaptada del cálculo hipergeométrico desarrollado por Lafon (1980) y de la metodología de análisis factorial con técnicas de correlación y aplicación de modelos de regresión multivariante (Larson-Hall, 2010). El cálculo hipergeométrico permite determinar la especificidad positiva por frecuencia de uso en los textos. Consideramos que existe especificidad positiva en la escritura digital síncrona, para aquellas formas utilizadas más de lo previsible, si se distribuyeran aleatoriamente en todo el corpus con un porcentaje superior al 13\% de los casos; y de especificidad negativa para las que se emplean en un índice menor al $13 \%$ en relación a su presencia aleatoria en el corpus global. El procedimiento ha consistido en calcular las desviaciones ortotipográficas y de inserción de elementos paralingüísticos (icónicos y audiovisuales) cada cien palabras. Para ello, hemos tomado como referencia los modelos estructurales de errores ortográficos de Backhoff, Peon, Andrade y Rivera (2008) y las principales categorías utilizadas en diversas investigaciones relacionadas con la ortografía del español (Bolet, 1999; 
Díaz, 2001; González, 1998; Mesanza, 1990; Pujol, 1999) que estipulan cocientes similares de error en estudiantes de edades comprendidas entre 13 y 16 años.

Ha habido distintas aproximaciones estadísticas al estudio de las especificidades, que se han basado en distribuciones teóricas tales como la de chi-cuadrado, la ley normal o la ley de Poisson. Sin embargo, es la ley hipergeométrica la que se adapta con exactitud a la población discreta de ocurrencias del vocabulario. Para el cálculo hipergeométrico de la especificidad, hemos recurrido a la identificación de los errores ortotipográficos y de empleo de elementos audiovisuales por medio del programa ATLAS.ti 7.0 en su aplicación Word Cruncher, para posteriormente hacer el volcado del texto plano en el programa Excel. Esto nos ha permitido identificar el porcentaje de aparición de palabras en las unidades textuales y poder calcular la distribución media de cada término en el conjunto de textos con un intervalo de confianza del $\mathrm{z}=1,96$. Asimismo, la aplicación de técnicas de análisis factorial, especialmente la correlación de Pearson y la generación de un modelo regresión lineal multivariante, nos ofrece la posibilidad de establecer patrones de uso de la escritura digital conforme a variables independientes que pueden condicionar el uso de este tipo de programas. Para el análisis factorial hemos empleado el programa SPSS 19.0. Para el análisis de correlación hemos codificado en el estudio seis variables independientes expuestas en la Tabla II.

Tabla II. Variables codificadas en el estudio.

\begin{tabular}{|l|c|c|}
\hline \multicolumn{1}{|c|}{ Variables } & \multicolumn{2}{c|}{ Subcomponentes } \\
\hline Sexo & Hombre & Mujer \\
\hline Edad & $13 / 15$ & $16 / 17$ \\
\hline Tamaño de la pantalla en pulgadas & $\geq 3$ pulgadas & $\leq 3$ pulgadas \\
\hline Ubicación de la conversación & Sin movimiento & En movimiento \\
\hline Horas de conversación & $\geq 1$ hora & $\leq 1$ hora \\
\hline $\begin{array}{l}\text { Relación entre interlocutores } \\
\text { (familia-amigos) }\end{array}$ & Familia & Amigos \\
\hline
\end{tabular}

Posteriormente, esta codificación se exportó en formato .txt y se creó el archivo en Excel que se empleó para el análisis estadístico cuantitativo en el programa SPSS. La frecuencia de palabras correspondiente a cada variable dependiente se ha realizado atendiendo a la siguiente fórmula:

$$
L w=\sum_{\substack{R \max \\ x=1 \\ T}} n\left(x^{L}\right) l_{n}\left(r_{\max }-r_{x}\right)
$$


En esta fórmula, $L w$ es la especificidad/densidad, $l$ es el porcentaje de lexemas y grafías de cada texto, $r_{\text {max }}$ es el rango con menor frecuencia y $r_{x}$ es el número de palabras en cada texto. $N_{x}$ es el número de palabras en el texto con rango $x$ y $T$ es el número total de palabras del texto. Dado que el porcentaje de lexemas y grafías es significativo con respecto al total de grafías y lexemas de los textos analizados, esta ecuación es significativa y apropiada para el análisis de longitud y frecuencia textual. Los resultados de las palabras, grafías, imágenes y enlaces requirieron, posteriormente, la utilización de un marco porcentual significativo que pudiera ser sometido a análisis cuantitativo y factorial en el programa SPSS.

Las variables independientes codificadas son fundamentales para el desarrollo de un análisis factorial que nos permita observar la influencia de las mismas en la producción escrita digital, síncrona y ubicua. De las posibles correlaciones y de la explicitación de un modelo de regresión sólido, se podrán obtener conclusiones relevantes que nos permitan delimitar las características y condicionantes de la escritura digital en este tipo de programas de mensajería.

\section{RESULTADOS Y DISCUSIÓN}

Los resultados se presentan atendiendo a los objetivos de la investigación en sus tres dimensiones:

\section{Caracterización y variación ortotipográfica de la escritura digital en WhatsApp}

Del total de 101401 palabras se ha procedido a realizar la identificación de las formas gráficas diferentes a la escritura normal por medio de la opción Word Cruncher del programa ATLAS.ti 7.0. Las formas distintas representan porcentualmente el 37,23\% del total de palabras. Las principales características lexicométricas de los textos codificados se muestran en la Tabla III, donde representamos la diferenciación entre casos y el total de palabras asociadas a esos casos con sus porcentajes respectivos.

Tabla III. Elementos ortotipográficos de la escritura digital en WhatsApp.

\begin{tabular}{|l|r|r|r|r|}
\hline $\begin{array}{c}\text { Dimensión 1: Caracterización ortotipográfica } \\
\text { de la escritura digital en WhatsApp }\end{array}$ & $\begin{array}{c}\text { Casos/ } \\
\text { Citas }\end{array}$ & \% Casos & Nb Words & $\%$ Words \\
\hline 1.1. Omisión absoluta de tildes & 32134 & $31,69 \%$ & 58230 & $57,42 \%$ \\
\hline 1.2. Unión gráfica de letras & 29567 & $29,15 \%$ & 43998 & $43,39 \%$ \\
\hline 1.3. Ortografía fonética/heterografías & 18761 & $18,50 \%$ & 31565 & $31,12 \%$ \\
\hline 1.4. Apócope y síncopa & 29876 & $29,46 \%$ & 32345 & $31,89 \%$ \\
\hline
\end{tabular}


Continuación Tabla III.

\begin{tabular}{|c|c|c|c|c|}
\hline 1.5. Errores de ortografía de la letra & 6790 & $6,69 \%$ & 9786 & $9,65 \%$ \\
\hline 1.6. Uso de mayúsculas & 1256 & $1,23 \%$ & 3471 & $3,42 \%$ \\
\hline 1.7. Signos matemáticos con valor fonológico & 234 & $0,23 \%$ & 541 & $0,53 \%$ \\
\hline 1.8. Omisión de preposiciones & 21 & $0,02 \%$ & 179 & $0,17 \%$ \\
\hline 1.9. Omisión de conjunciones & 72 & $0,07 \%$ & 213 & $0,21 \%$ \\
\hline 1.10. Omisión de artículos & 38 & $0,03 \%$ & 183 & $0,18 \%$ \\
\hline 1.11. Presencia de signos de puntuación & 1789 & $1,76 \%$ & 2134 & $2,10 \%$ \\
\hline 1.12. Duplicaciones de letras & 1234 & $1,21 \%$ & 3189 & $3,14 \%$ \\
\hline 1.13. Aféresis vocales & 134 & $0,13 \%$ & 241 & $0,23 \%$ \\
\hline 1.14. Aféresis consonantes & 123 & $0,12 \%$ & 199 & $0,19 \%$ \\
\hline 1.15. Epéntesis & 34 & $0,03 \%$ & 45 & $0,04 \%$ \\
\hline TOTAL & 123041 & & 187644 & \\
\hline
\end{tabular}

A continuación presentamos la muestra de especificidad más representativa en las subdimensiones analizadas correspondientes a la ortotipografía de la escritura en WhatsApp.

Tabla IV. Ejemplos y coeficientes de especificidad en la ortotipografía de la escritura digital en WhatsApp.

\begin{tabular}{|c|c|c|}
\hline Ortotipografía & Ejemplos en textos & $\begin{array}{l}\text { Coeficiente de } \\
\text { especificidad }\end{array}$ \\
\hline $\begin{array}{l}\text { 1.1. Omisión absoluta } \\
\text { de tildes }\end{array}$ & $\begin{array}{l}\text { a y yo no te decia nah cunado me decian q estavas } \\
\text { todos los dias ayy tonteando con carlos agudo... Ino } \\
\text { si se ve q te a venido mejor... tanto q sigues picaa }\end{array}$ & $\mathrm{N}=98785 / 93 \%$ \\
\hline $\begin{array}{l}\text { 1.2. Unión gráfica de } \\
\text { letras }\end{array}$ & $\begin{array}{l}\text { mi padre que me ha castigado sin ir asik por eso no } \\
\text { voy jajal A las } 7 \text { imedia tengo spining/Aver si van a } \\
\text { entrar o algo/Astamañana. }\end{array}$ & $\mathrm{N}=2341 / 43 \%$ \\
\hline $\begin{array}{l}\text { 1.3. Ortografía } \\
\text { fonética/heterografías }\end{array}$ & $\begin{array}{l}\text { Sii } k \text { as desaparecido. Parece que no kieres } k \text { t la } \\
\text { del Aiba se me a olvidadolperdon x ser pesadol si } \\
\text { a dixoq no qiere nah con nadiel Ps yastal asiesque } \\
\text { bien }\end{array}$ & $\mathrm{N}=3341 / 56 \%$ \\
\hline 1.4. Apócope y síncopa & $\begin{array}{l}\text { No pasa nal Y pa saber si te lo pagol yo lo mismo } \\
\text { te puedo llevar con un colega o algo sabsl jaja nos } \\
\text { las ganams un poco y quedms cn ellasl y dime has } \\
\text { hablao con estas. }\end{array}$ & $\mathrm{N}=3456 / 57 \%$ \\
\hline $\begin{array}{l}\text { 1.5. Errores de } \\
\text { ortografía de la letra }\end{array}$ & $\begin{array}{l}\text { haber si un dia me habisas y voy al gym a por la } \\
\text { mesa no hay prisa pero cuando la lleves habisame } \\
\text { jaja } \\
\text { haber si mañana voy a por ella } \\
\text { Yo cuando me vaya me voy a poner to depre }\end{array}$ & $\mathrm{N}=2789 / 51 \%$ \\
\hline
\end{tabular}


RLA. Revista de Lingüística Teórica y Aplicada, 53 (1), I Sem. 2015

Continuación Tabla IV.

\begin{tabular}{|c|c|c|}
\hline 1.6. Uso de mayúsculas & $\begin{array}{l}\text {... eso es tratar bien a las muxaxas... y si es verdad } \\
\text { estava tan rayao q no sentia por ti lo mismo, y yo te } \\
\text { pedi un tiempo.. te recuerdo q fuistes tu qien dijo q } \\
\text { si te dejaba era pa siempree. }\end{array}$ & $\mathrm{N}=987 / 89 \%$ \\
\hline $\begin{array}{l}\text { 1.7. Signos matemáticos } \\
\text { con valor fonológico }\end{array}$ & $\begin{array}{l}\text { no tengo sus numero tenia el de } 2 \text { que estaban hay/ } \\
\text { bien soy prestigio } 1 \text { y tu??/Salu2 }\end{array}$ & $\mathrm{N}=356 / 7 \%$ \\
\hline $\begin{array}{l}\text { 1.8. Omisión de } \\
\text { preposiciones }\end{array}$ & Tuu as ido Ø por las fundas?? & $\mathrm{N}=78 / 0,1 \%$ \\
\hline $\begin{array}{l}\text { 1.9. Omisión de } \\
\text { conjunciones }\end{array}$ & $\begin{array}{l}\text { Si me llevan sii Ø yo tener lo voy. Atener pero el } \\
\text { saber cuando me lleven no lo se asik az lo que tu } \\
\text { veas que es mejor jaja }\end{array}$ & $\mathrm{N}=89 / 0,3 \%$ \\
\hline $\begin{array}{l}\text { 1.10. Omisión de } \\
\text { artículos }\end{array}$ & voy a ver si vendo $\varnothing$ bb y si la vendo, te lo pillo va? & $\mathrm{N}=99 / 0,4 \%$ \\
\hline $\begin{array}{l}\text { 1.11. Presencia de } \\
\text { signos de puntuación }\end{array}$ & $\begin{array}{l}\text { No solo puedo ir yo con otro chaval al sitio ese } \\
\text { porque donde voy no puede ir nada mas y tengo } \\
\text { que ir a villalba }\end{array}$ & $\mathrm{N}=708 / 92 \%$ \\
\hline $\begin{array}{l}\text { 1.12. Duplicaciones de } \\
\text { letras }\end{array}$ & $\begin{array}{l}\text { Valee tochoo. Tuuu no faltes mañana. Vamoss } \\
\text { kikee. Tu di de quedar. ya se lo e dixooo jajajja. } \\
\text { POOOORRRRRRSUPUESTO. Assi me gussta } \\
\text { máquina. Yo noo tioo. Joodrr. A. }\end{array}$ & $\mathrm{N}=2438 / 62 \%$ \\
\hline 1.13. Aféresis vocales & $\begin{array}{l}\text { jaja nos las ganams un poco y quedms cn ellas/ Yo } \\
\text { ete finde te digo algo }\end{array}$ & $\mathrm{N}=1654 / 53 \%$ \\
\hline $\begin{array}{l}\text { 1.14. Aféresis } \\
\text { consonantes }\end{array}$ & ami me eengaa con las cosas claras & $\mathrm{N}=1438 / 52 \%$ \\
\hline 1.15. Epéntesis & Sip/Valep, perfect & $\mathrm{N}=538 / 23 \%$ \\
\hline
\end{tabular}

$\mathrm{N}=$ número total de casos documentados.

$\%=$ porcentaje de especificidad por muestra aleatoria en textos.

Como se puede observar en los ejemplos con mayor especificidad, es significativo:

- La omisión casi absoluta de las tildes.

- La escritura oralizada fonéticamente. Así letras como "k", "d", "t" pasan a representar sílabas al leer el nombre de la letra y no su valor en el sistema ortográfico español.

- La unión de palabras.

- La tendencia a la falta de ortografía con la letra "h".

- Tendencia a la supresión de vocales (apócope y síncopa).

- La ausencia casi generalizada de mayúsculas con valor ortográfico y de signos de puntuación. 
- El empleo de mayúsculas con valor pragmático de exclamación y resalte interpretativo.

Existe una especificidad baja y, por lo tanto, son aspectos poco significativos en comparación con estudios previos de escritura con SMS (Jaffe y Walton, 2000; Cassany, 2003; Bodomo y Lee, 2004; Betti, 2006), los siguientes:

- Uso reducido de números y signos matemáticos con valor fonológico.

- No hay el porcentaje de reducción de caracteres para acumular más información.

- No existe el porcentaje de omisión de determinados conectores (artículos, preposiciones, conjunciones, adverbios, etc.) y en general de las palabras que no se consideran imprescindibles para la comunicación, que se documenta en los SMS.

- Menor acortamiento en monosílabos.

2. Materialización de los elementos gráficos para la representación suprasegmental y elementos audiovisuales como apoyo al discurso escrito

Del total de 101401 palabras, se ha procedido a realizar la identificación de las formas gráficas con representación suprasegmental y los elementos audiovisuales que complementan la escritura digital; para ello, utilizamos la opción de búsqueda de frecuencias del programa ATLAS.ti 7.0 mediante búsqueda de imagen y duplicación de caracteres de materialización de la risa, emoticonos y "stickers". Las formas gráficas (emoticonos fijos, en movimiento y "stickers") con interpretación suprasegmental representan porcentualmente el 17,31\% del total de signos gráficos presentes en los textos digitales; lo que supone un porcentaje bastante alto. Las principales características suprasegmentales y audiovisuales se presentan en las tablas V y VI.

Tabla V. Elementos gráficos con interpretación suprasegmental.

\begin{tabular}{|c|c|c|c|}
\hline $\begin{array}{l}\text { Dimensión 2: Elementos gráficos con } \\
\text { interpretación suprasegmental }\end{array}$ & $\%$ Casos & Total No & \% Especificidad \\
\hline Emoticonos fijos & $10,1 \%$ & 1390 & $39,6 \%$ \\
\hline Emoticonos en movimiento & $4,9 \%$ & 567 & $28,3 \%$ \\
\hline Stickers & $2,3 \%$ & 159 & $17,1 \%$ \\
\hline TOTAL & $17,31 \%$ & 2116 & $+37 \%$ \\
\hline Risa & $59,3 \%$ & 8934 & $57,1 \%$ \\
\hline
\end{tabular}


Los elementos gráficos con interpretación suprasegmental han aumentado al hacerse los emoticonos muy elaborados e incorporar imágenes e iconos denominados "stickers" (representaciones icónicas o de personajes relevantes de películas o de la actualidad social, generalmente con una interpretación irónica o humorística). Todos estos elementos gráficos de los programas de mensajería representan un abanico muy grande de estados de ánimo y emociones. El porcentaje de especificidad en cada conversación es bastante relevante y alcanza el 37\% de la muestra de mensajes. Se han contabilizado un total de 1390 emoticonos estáticos, 567 emoticonos en movimiento y 159 "stickers" que se insertan en los mensajes, principalmente al finalizar una intervención escrita (95\%). Su frecuencia de uso es mayor en conversaciones con amigos $(+34 \%)$ que con la familia. Asimismo, la materialización de la risa sigue siendo un elemento recurrente de todos los mensajes cortos y, con la no limitación de caracteres, se ha aumentado su frecuencia en cada conversación digital. La interpretación pragmática de la misma no corresponde siempre con la materialización del gesto, sino con una muestra de amistad y un elemento fático que refuerza el sentimiento compartido. La categorización de la risa se estableció en relación a la repetición de onomatopeyas lingüísticas, principalmente: "jeje” o "jaja”; y por el empleo de elementos icónicos (stickers o emoticonos con caras sonrientes). Su materialización mayor suele ser la repetición de dos o tres "jajaja” o "jejeje”. Su incorporación es más común al final (79\%) o al comienzo (21\%) de una intervención y se suele realizar más con amigos $(41 \%)$ que con familiares (19\%). Su coeficiente de especificidad es muy alto $(57,1 \%)$; lo que implica que es un recurso de interpretación fática y de refuerzo de la amistad en el discurso digital.

En la Tabla VI se presenta los coeficientes de aparición y especificidad de los elementos audiovisuales, que sirven de apoyo al desarrollo del discurso escrito digital.

Tabla VI. Elementos audiovisuales como complemento a la escritura digital.

\begin{tabular}{|c|c|c|c|}
\hline $\begin{array}{l}\text { Dimensión 3: Elementos audiovisuales } \\
\text { como complemento a la escritura digital }\end{array}$ & $\begin{array}{c}\% \\
\text { Casos }\end{array}$ & Total No & $\%$ Especificidad \\
\hline Imagen & $27,9 \%$ & 6734 & $29,8 \%$ \\
\hline Video & $13,1 \%$ & 3067 & $19,1 \%$ \\
\hline Sonido & $5,9 \%$ & 567 & $4,3 \%$ \\
\hline Enlaces a video & $10,3 \%$ & 1121 & $11,3 \%$ \\
\hline TOTAL & $57,2 \%$ & 11489 & $31 \%$ \\
\hline
\end{tabular}

La diferencia mayor con respecto a los anteriores SMS (Bodomo y Lee, 2004; Betti, 2006) es la incorporación de elementos audiovisuales a la escritura digital, 
como refuerzo del mensaje lingüístico y como forma de complementar pragmáticamente lo que se quiere decir o comunicar. Este tipo de funcionalidades convierten a la escritura digital en un poderoso elemento de comunicación que nos permite la visualización de imagen, video y la referencia intertextual a otros textos o formatos audiovisuales de Internet. Se ha diferenciado en la investigación entre el envío de archivos de datos y el envío de enlaces a contenido en la Red. La diferencia estriba en que la imagen, video o sonido archivado ha sido previamente visualizado y considerado pertinente por el interlocutor y su remisión suele ser más rápida que la visualización de enlaces. Asimismo, estos archivos se remiten principalmente para iniciar una conversación o contacto $(35,7 \%)$ o para complementar lo comentado o transmitido en una conversación (41\%). Los archivos con mayor porcentaje de remisión son la imagen personal $(36 \%)$ y la imagen social (21\%); el video social (17\%) y el video personal (10\%). Se suelen remitir más imágenes personales $(21 \%)$ que sociales (18\%) o gráficos y más videos de terceras personas $(15 \%)$ que personales $(11 \%)$. La remisión de enlaces es significativamente menor al envío de archivos (-23\%), probablemente porque el contenido en la Red es más lento de visualizar y dificulta la inmediatez en el desarrollo de las conversaciones.

\section{Correlaciones entre variables y modelo de regresión lineal multivariante explicativo}

El modelo de regresión lineal propuesto permite establecer una relación matemática entre el conjunto de las seis variables independientes codificadas en la investigación, denominadas como covariantes o factores, y las variables dependientes analizadas: variación ortotipográfica, elementos gráficos y audiovisuales de la escritura digital. De esta manera, la ecuación que se propone a continuación puede funcionar como modelo predictivo de la escritura digital en este tipo de programas y servir, la significación obtenida, como factor explicativo en las implicaciones sociales, lingüísticas y pedagógicas. Una vez analizado este modelo, se postulará la ecuación que genera mayor porcentaje de explicación de las características de la escritura digital entre adolescentes. El modelo propuesto es el siguiente:

$$
\begin{aligned}
& y i j k=\beta 0 j k+\beta 1 E D i j k+\beta 2 \text { SEijk }+\beta 3 P U L i j k++\beta 4 U B \mathrm{ij} k+\beta 5 H C \mathrm{i} j k+\beta 6 R F i j k \\
& +\beta 7 R A \mathrm{i} j k+\varepsilon i j k \\
& \beta 0 \mathrm{i} j k=\beta 0+v 0 k+\mu 0 k \\
& \beta 1 \mathrm{ij} k=\beta 1+v 1 k+\mu 1 k \ldots \beta 4 j k=\beta 4+v 4 k+\mu 4 k \ldots \\
& \text { Con: } \\
& {[\varepsilon 0 i j k]-N(0, \Omega \varepsilon): \Omega \varepsilon=[\sigma 2 \varepsilon 0]} \\
& {[\mu 0 j k]-N(0, \Omega \mu): \Omega \mu=[\sigma 2 \mu 0]} \\
& {[v 0 k]-N(0, \Omega v): \Omega v=[\sigma 2 v 0]}
\end{aligned}
$$


Donde las variables dependientes son: $i=$ desviación ortotipográfica, $j=$ elementos suprasegmentales-gráfico y $k=$ elemento audiovisual (yijk: variables dependientes).

Y las variables independientes:

NEDijk, edad del estudiante.

NSEijk, sexo del estudiante.

PULijk, tamaño de la pantalla en pulgadas.

UBijk, ubicación de la conversación.

HCijk, horas de conversación.

$R$ Fijk, relación entre interlocutores (familia).

RAijk, relación entre interlocutores (amigos).

En la Tabla VII se presenta el resumen del modelo de regresión lineal y se realiza el análisis de correlaciones según el modelo ecuacional propuesto, tomando como constantes las tres variables independientes del modelo: "modelo 1": ortotipografía, "modelo 2": elementos gráficos y "modelo 3": elementos audiovisuales.

Tabla VII. Resumen del modelo de regresión lineal multivariante de la escritura digital en WhatsApp.

\begin{tabular}{|l|c|c|c|c|}
\hline & Resumen del modelo \\
\hline Modelo & $\mathrm{R}$ & R cuadrado & $\begin{array}{c}\text { R cuadrado } \\
\text { corregida }\end{array}$ & $\begin{array}{c}\text { Error típ. de la } \\
\text { estimación }\end{array}$ \\
\hline 1 &, $389^{\mathrm{a}}$ &, 240 &, 210 & 15,430287 \\
2 &, $235^{\mathrm{b}}$ &, 142 &, 112 & 15,185607 \\
3 &, $268^{\mathrm{d}}$ &, 135 &, 135 & 14,989680 \\
\hline
\end{tabular}
a. Variables predictoras: (constante), ortotipografía de la escritura en WhatsApp.
b. Variables predictoras: (constante), elementos gráficos con interpretación suprasegmental.
c. Variables predictoras: (constante), elementos audiovisuales como complemento a la escritura digital.

El modelo de la escritura digital analizado explica el 51,7\% de las variaciones de este tipo de escritura con respecto a la lengua estándar. El "modelo 1" de variación ortotipográfica es el que más varianza total explica (24\%). El "modelo 2" de elementos gráficos de interpretación suprasegmental (elementos icónicos) explica el 14,2\% de la varianza total y el "modelo 3" de elementos audiovisuales como complemento a la escritura digital explica el 13,5\%, respectivamente. En la 
Tabla VIII se presenta los resultados de coeficientes del modelo general propuesto, donde se pueden ver los resultados de incidencia de las variables independientes, en cada una de las variables dependientes analizadas.

Tabla VIII. Resultados de coeficientes del modelo.

\begin{tabular}{|c|c|c|c|c|c|c|}
\hline \multicolumn{7}{|c|}{ Coeficientes $^{\text {abc }}$} \\
\hline & & \multicolumn{2}{|c|}{$\begin{array}{c}\text { Coeficientes no } \\
\text { estandarizados }\end{array}$} & \multirow{2}{*}{$\begin{array}{c}\begin{array}{c}\text { Coeficientes } \\
\text { tipificados }\end{array} \\
\text { Beta }\end{array}$} & \multirow[b]{2}{*}{$\mathrm{t}$} & \multirow[b]{2}{*}{ Sig. } \\
\hline \multicolumn{2}{|c|}{ Modelo } & B & Error típ. & & & \\
\hline \multirow[t]{8}{*}{1} & (Constante) & 60,575 & ,491 & & 123,484 &, 000 \\
\hline & Sexo & 4,467 & , 187 & ,277 & 23,838 &, 567 \\
\hline & Edad & 3,611 & , 193 & , 167 & 14,476 & ,411 \\
\hline & Pulgadas & 2,011 & ,200 &, 116 & 10,792 &, 000 \\
\hline & Ubicación & 2,751 &, 127 & ,089 & 14,476 &, 000 \\
\hline & Horas & 2,771 & , 113 & , 167 & 10,792 &, 000 \\
\hline & Amigos & 2,652 & , 111 & ,116 & 14,476 &, 000 \\
\hline & Familia & 2,869 &, 175 & ,171 & 14,672 &, 000 \\
\hline \multirow[t]{8}{*}{2} & (Constante) & 53,633 & ,806 & & 66,507 & ,000 \\
\hline & Sexo & 4,366 & , 186 & ,271 & 23,467 &, 541 \\
\hline & Edad & 3,611 & , 193 & , 167 & 14,476 & ,410 \\
\hline & Pulgadas & 2,011 & ,200 &, 116 & 10,792 &, 000 \\
\hline & Ubicación & 2,751 &, 127 & ,089 & 14,476 &, 000 \\
\hline & Horas & 2,771 &, 113 & , 167 & 10,792 &, 000 \\
\hline & Amigos & 2,807 & , 194 & , 167 & 14,476 &, 000 \\
\hline & Familia & 2,162 & ,200 &, 124 & 10,792 &, 000 \\
\hline \multirow[t]{8}{*}{3} & (Constante) & 49,760 & ,950 & & 52,364 &, 000 \\
\hline & Sexo & 4,167 & , 187 & ,259 & 22,280 & ,499 \\
\hline & Edad & 2,813 & 193 & 167 & 14,571 & ,398 \\
\hline & Pulgadas & 2,014 & ,200 & , 116 & 10,048 &, 000 \\
\hline & Ubicación & 2,857 & ,375 & ,089 & 7,619 &, 000 \\
\hline & Horas & 2,813 & , 193 & , 167 & 14,571 & ,000 \\
\hline & Amigos & 2,014 & ,200 & ,116 & 10,048 & ,000 \\
\hline & Familia & 2,857 & 375 & ,089 & 7,619 & ,000 \\
\hline
\end{tabular}

Modelo 1. Variable dependiente: ortotipografía de la escritura en WhatsApp.

Modelo 2. Variable dependiente: elementos gráficos con interpretación suprasegmental.

Modelo 3. Variable dependiente: elementos audiovisuales como complemento a la escritura digital. 
De las seis variables independientes analizadas en el modelo, han resultado altamente significativas (Sig. ,000; correlación positiva) las siguientes: "pulgadas del smartphone" (altamente significativa en el modelo 1 "Variación ortotipográfica"). Este resultado implica que cuando los smartphones disponen de pantallas inferiores a 4 pulgadas, se produce un mayor número de errores ortotipográficos por el tamaño del teclado táctil $(+31,5 \%)$. La "ubicación de la conversación" es altamente significativa en los tres modelos; cuando la conversación se realiza en movimiento, se comete un mayor número de errores ortotipográficos $(+41,3 \%)$ y se remite un número menor de elementos gráficos y audiovisuales $(-21,6 \% /-18,3 \%)$. La variable "horas de conversación" también es altamente significativa en los tres modelos. Aquellos usuarios que hacen uso del WhatsApp más de una hora al día cometen más errores ortotipográficos, especialmente heterografías $(+22,1 \%)$ y remiten más elementos gráficos y audiovisuales $(+16,3 \%)$. La "conversación con amigos y en familia" no es significativa para el "modelo 1 " pero sí es altamente significativa para las variables dependientes b y c ("modelos 2 y 3 ”). Se remiten más elementos gráficos $(+15,3 \%)$ y audiovisuales $(+12,1 \%)$ en las conversaciones con amigos que entre familiares. Han resultado no significativas las variables independientes sexo (Sig. ,501 de media en las tres variables) y edad (Sig. ,403 de media en las tres variables), por lo que ambas variables no influyen en las tres variables dependientes analizadas. Por lo tanto, el modelo de regresión lineal multivariante queda configurado con la siguiente ecuación, que explica el 51,7\% de la variabilidad y especificidad de la escritura digital en WhatsApp, con respecto a las variables dependientes analizadas: errores ortotipográficos, elementos gráficos y audiovisuales empleados en la escritura digital en WhatsApp.

$$
y i j k=\beta 0 j k+\beta 3 P U L i j k++\beta 4 U B \mathrm{i} j k+\beta 5 H C \mathrm{i} j k+\beta 6 R F i j k+\beta 7 R A \mathrm{i} j k+\varepsilon i j k
$$

$y i j k$, son las diferentes variables dependientes ( $i$ : ortotipografia; $j$ : elementos gráficos; $k$ : elementos audiovisuales).

PULijk, tamaño de la pantalla en pulgadas.

$U B \mathrm{i} j k$, ubicación de la conversación.

$H C \mathrm{i} j k$, horas de conversación.

$R F_{i j k}$, relación entre interlocutores (familia).

$R A \mathrm{ijk}$, relación entre interlocutores (amigos).

\section{CONCLUSIONES}

La escritura digital preside nuestras vidas y nuestras relaciones personales, académicas y profesionales. Entre los adolescentes, la escritura digital por medio de 
programas de mensajería tipo WhatsApp se ha convertido en uno de los instrumentos fundamentales de comunicación diaria. Las repercusiones lingüísticas, pedagógicas y sociales de la escritura digital precisan de estudios que puedan suscitar y reflexionar sobre las posibles implicaciones en la competencia social, digital y lingüística de las personas. Los objetivos de esta investigación han tenido una doble vertiente. En primer lugar, documentar la variabilidad de la escritura digital conforme a tres variables dependientes: variación ortotipográfica, empleo de elementos gráficos con interpretación suprasegmental (elementos icónicos) y uso de elementos audiovisuales como complemento a la escritura digital; en segundo lugar, hemos pretendido conformar un modelo explicativo de la escritura digital de forma que pueda ser validado o contrastado en otros estudios o investigaciones.

Los resultados han revelado cómo la escritura digital en este tipo de programas es sustancialmente diferente a la que se realizaba hace unos años en los tradicionales SMS de 140 caracteres. La variación ortotipográfica es menor y se ve menos afectado el acortamiento de las palabras. Los elementos de variación ortotipográfica más relevantes son: la ausencia de mayúsculas y tildes, la duplicación de letras, la unión de palabras, la ortografía fonética, las heterografías y los errores en la ortografía de las letras (principalmente " $h$ "). El hecho más relevante de la escritura digital en WhatsApp es el enriquecimiento suprasegmental por un variado tipo de grafismo fijo y en movimiento con emoticonos, "stickers" y la materialización de la risa como elemento fático y de cortesía digital. Junto con esta representación gráfica de lo paralingüístico, de las emociones y estados de ánimo, la escritura digital se complementa con un elemento hasta ahora nunca incorporado en el smartphone, los elementos audiovisuales en formato de archivo o hiperenlace que facilitan la conexión de datos de estos dispositivos móviles y que enriquecen el discurso escrito como nunca antes se había realizado. La imagen, video y sonido que puede acompañar al texto convierte a las conversaciones digitales en un medio de comunicación altamente productivo que integra casi todos los medios de representación de la realidad en un discurso digital ciberlingüístico.

\section{LIMITACIONES Y PROSPECTIVA}

El estudio se ha contextualizado en España y su incidencia responde a la variedad del español peninsular. Sería deseable que futuros estudios contextualizados en otras zonas de habla hispana complementaran las conclusiones aquí presentadas. Asimismo, el análisis comparativo entre diferentes lenguas podría suscitar nuevas interpretaciones que aportarán seguro nuevas formas de interpretar la escritura digital síncrona y ubicua. Este estudio se ha centrado, principalmente, en el análisis lexicométrico de los ámbitos lingüístico y paralingüístico, como paso previo 
a futuros estudios, en los que otras áreas como la sociolingüística o la didáctica tendrán mucho que aportar.

\section{REFERENCIAS}

Alonso, E. y Perea, M. (2008). SMS: Impacto social y cognitivo. Escritos de Psicología, 2 (1), 24-31.

Andrade, L. (2008). Los SMS: nuevas formas de interacción juvenil. Tesis doctoral. Facultad Latinoamericana de Ciencias Sociales, Ecuador: Quito.

Backhoff, E., Peon, M., Andrade, E. y Rivera, S. (2008). La ortografía de los estudiantes de educación básica en México. México: Instituto nacional para la evaluación de la educación.

Bañon, A. (2010). Las nuevas tecnologías y la comunicación de jóvenes y adolescentes. Usos y discursos. Interacçōes, 16, 64-89.

Baron, N., Squires, L., Tench, S. y Thompson, M. (2005). Tethered or mobile? Use of away messages in instant messaging by American college students. En Mobile Communications: Re-Negotiation of the Social Sphere, ed. by Richard Ling \& Per E. Pedersen (pp. 293-311). London: Springer-Verlag.

Benzécri, J.P. (1973). L’Analyse des données. Paris: Dunod.

Benzécri, J.P. (1979). Sur le calcul des taux d'inertie dans l'analyse d'un questionnaire. Cahiers de l'Analyse des Données, 4, 377-378.

Bernicot, J., Goumi, A., Bert-Erboul, A. y Volckaert-Legrier, O. (2014). How do skilled and less-skilled spellers write text messages? A longitudinal study. Journal of Computer Assisted Learning, 30, 559-576.

Betti, S. (2006). La jerga juvenil de los SMS. Cuadernos del Lazarillo, 31, 68-76.

Bodomo, A.B. y Lee, C.K.M. (2004). Linguistic features of SMS texts in Hong Kong. Australian Journal of Communication, 11, 63-85.

Bolet, F.J. (1999). Errores de tildación en la redacción de textos narrativos y expositivos. Revista Paradigma, 20 (2), 133-144.

Cassany, D. (2003). La escritura electrónica. Cultura y educación, 3 (15), 239-251.

Castells, M. (2007). La transición a la sociedad Red. Barcelona: Ariel.

Castells, M. (2009). Comunicación y poder. Madrid: Alianza Editorial.

Crystal, D. (2008). Txtng: the Gr8 D68. Oxford: Oxford University Press.

Díaz, C. (2001). Constancia y variación gráfica en la evaluación conceptual de la escritura: una aproximación a la comprensión del conocimiento ortográfico. Tesis de Doctorado, México: Centro de Investigación y de Estudios Avanzados del Instituto Politécnico Nacional. Departamento de Investigaciones Educativas.

Dresner, E. y Herring, S.C. (2012). Emoticons and illocutionary force. En D. Riesenfel \& G. Scarafile (Eds.), Philosophical dialogue: Writings in honor of Marcelo Dascal (pp. 59-70). London: College Publication. 
Drouin, M. (2011). College students' text messaging, use of textese and literacy skills. Journal of Computer Assisted Learning, 27, 67-75.

Elvis, F.W. (2009). The Sociolinguistics of mobile phone SMS usage in Cameroon and Nigeria. The International Journal of Language Society and Culture, 28 (28), 25-41.

Etxeberría, J., García, E., Gil J. y Rodríguez, G. (1995). Análisis de datos y textos. Madrid: RA-MA Ediciones.

Faulkner, X. y Culwin, F. (2005). When fingers do the talking: a study of text messaging. Interacting with Computers, 17 (2), 167-185.

Fernández Pinto, J. (2001). Apuntes sobre SMS y WAP. Cuadernos Cervantes, $1,76-85$.

Flores Vivar, J.M. (2009). Nuevos modelos de comunicación, perfiles y tendencias en las redes sociales. Comunicar, 33, 73-81.

Fortunati, L. (2001). The mobile phone between orality and writing. Third International Conference on Uses and Services in Telecommunications. París.

Fraca de Barrera, L. (2006). La ciberlingua. Una variedad compleja de lengua en Internet. Caracas: Instituto Venezolano de Investigaciones Lingüísticas y Literarias "Andrés Bello".

Fundación Telefónica (2014). The App Date. Recuperado de http://madrid.theappdate.com/informe-sobre-las-apps-en-espana-2014-adictosa-la-mensajeria/\# (20 de septiembre de 2014).

García, Ma.C. y Monferrer, J. (2009). Propuestas de análisis teórico sobre el análisis del teléfono móvil en adolescentes. Comunicar, 33, 83-92.

García Galera, M.C. (2008). La telefonía móvil en la infancia y adolescencia. Usos, influencias y responsabilidades. Madrid: Proyecto de investigación financiado por la oficina del Defensor del Menor de la Comunidad de Madrid.

Gelernter, J. y Mushegian, N. (2011). Geo-parsing messages from microtext. Transactions in GIS, 15 (6), 753-773.

Gómez Camacho, A. (2007). La ortografía del español y los géneros electrónicos. Comunicar, 29, 157-164.

Gómez Torrego, L. (2001). La Gramática en Internet. Recuperado de: http://cvc.cervantes.es/obref/congresos/valladolid/ponencias/nuevas_ fronteras_del_espanol/4_lengua_y_escritura/gomez_l.htm (1 de septiembre de 2014).

González, J.A. (1998). Diagnosis ortográfica en contexto andaluz. Escuela abierta, 1, 127-194.

González-Lloret, M. (2011). Conversation Analysis of Computer-mediated communication. CALICO Journal, 28 (2), 308-325.

Herring, S.C. (2013a). Relevance in computer-mediated conversation. En 
S. C. Herring, D. Stein, \& T. Virtanen (Eds.), Handbook of pragmatics of computer-mediated communication (pp. 245-268). Berlin: Mouton.

Herring, S.C. (2013b). Discourse in Web 2.0: Familiar, reconfigured, and emergent. En D. Tannen \& A. M. Tester (Eds.), Georgetown University Round Table on Languages and Linguistics 2011: Discourse 2.0: Language and new media (pp. 1-25). Washington, DC: Georgetown University Press.

Horstmanshof, L. y Power, M.R. (2005). Mobile phones, SMS, and relationships. Australian Journal of Communication, 32 (1), 33-52.

Hutchby, I. y Tanna, V. (2008). Aspects of sequential organization in text message exchange. Discourse and Communication, 2 (2), 143-164.

Jaffe, A. y Walton, S. (2000). The voices people read: orthography and the representation of non-standard speech. Journal of Sociolinguistics 4 (4), 561-587.

Kamran, S. (2010). Mobile Phone: Calling and texting patterns of college students in Pakistan. International Journal of Business and Management, 5 (4), 26-36.

Kemp, N. (2010). Texting vs. txting: Reading and writing text messages, and links with other linguistic skills. Writing Systems Research, 2, 53-71.

Kuteeva, M. (2011). Wikis and Academic Writing: Changing the WriterReader Relationship. English for Specific Purposes, 30 (1), $44-57$.

Lafon, P. (1980). Sur la variabilité des fréquences des formes dans un corpus. Mots, $1,127-165$.

Larson-Hall, J. (2010). A Guide to Doing Statistics in Second Language Research Using SPSS. New York, NY: Routledge.

Lebart, L. y Salem, A. (1994). Statistique Textuelle. Paris: Dunod.

Lee, C.K.M. (2011). Micro-Blogging and Status Updates on Facebook: Texts and Practices. En Thurlow, Crispin/Mroczek, Kristine (eds.): Digital Discourse. Language in the New Media (pp. 110-128). Oxford/New York: Oxford University Press.

Lewis, C. y Fabos, B. (2005). Instant messaging, literacies, and social identities. Reading Research Quarterly, 40 (4), 470-500.

Mayans i Planells, J. (2002). De la incorrección normativa en los chats. Revista de investigación Lingüistica, 2 (5), 101-116.

McWilliam, L., Schepman, A. y Rodway, P. (2009). The linguistic status of text message abbreviations: An exploration using a Stroop task. Computers in Human Behavior, 25 (4), 970-974.

Markman, K.M. (2013). Conversational coherence in small group chat. En S. Herring, D. Stein, and T. Virtanen (Eds.), Pragmatics of Computer-Mediated Communication (pp. 539-564). Berlin: Mouton de Gruyter. 
Mesanza, J. (1990). Palabras que peor escriben los alumnos. Inventario cacográfico. Madrid, España: Editorial Escuela Española.

Nastri, J., Peña, J. y Hancock, J.T. (2006). The construction of away messages: A speech act analysis. Journal of Computer-Mediated Communication, 11 (4), 7-16.

Parrilla, E.A. (2008). Alteraciones del lenguaje en la era digital. Comunicar, 30, 131-136.

Pennebaker, J.W., Booth, R.J. y Francis, M.E. (2007). Linguistic Inquiry and Word Count (LIWC2007): A text analysis program. Austin, TX: LIWC.net.

Pinar, M.J. y Albentosa, J.I. (2001). Implicaciones lingüisticas del uso de mensajes de texto en teléfonos móviles. XXXI Simposio de la Sociedad Española de Lingüística. Universidad de Almería, Almería, 17-20 de diciembre de 2001.

Plester, B., Wood, C. y Bell, V. (2008). Text Msg in school literacy: Does texting and knowledge of text abbreviations adversely affect children's literacy attainment? Literacy, 42 (3), 137-144.

Plester, B., Wood, C. y Joshi, P. (2009). Exploring the relationship between children's knowledge of text message abbreviations and school literacy outcomes. British Journal of Developmental Psychology, 27 (1), 145-161.

Pujol, M. (1999). Análisis de errores grafemáticos en textos libres de estudiantes de enseñanzas medias. Tesis de Doctorado. Departamento de Didáctica de la Lengua y la Literatura. Universidad de Barcelona.

Riordan, M.A., Markman, K.M. y Stewart, C.O. (2013). Communication accommodation in instant messaging: An examination of temporal convergence. Journal of Language and Social Psychology, 32 (1), 84-95.

Rivière, C.A. (2002). La práctica del mini-mensaje en las interacciones cotidianas: una doble estrategia de exteriorización y de ocultación de la privacidad para mantener el vínculo social. Revista de estudios de juventud, 57, 125-137.

Rosen, L., Chang, J., Erwin, L., Carrier, L.M. y Cheever, N.A. (2010). The relationship between textisms and formal and informal writing among young adults. Communication Research, 37 (3), 420-440.

Treurniet, M. y Sanders, E. (2012). Chats, Tweets and SMS in the SoNaR Corpus: Social Media Collection. Proceedings 1st Annual International Conference Language, Literature \& Linguistics (L3 Conference) (pp. 268-271). Singapore: GSTF.

Vázquez-Cano, E. (2012). Mobile Learning with Twitter to Improve Linguistic Competence at Secondary Schools. The New educational Review, 29, (3), 134147.

Yus, F. (2011). Cyberpragmatics: Internet-Mediated Communication in Context. Amsterdam/Philadelphia: John Benjamins. 